\title{
Hyperprogressive disease in advanced cancer patients with liver metastasis treated with PD-1 inhibitors: two case reports
}

\author{
Zhuangbin Lin ${ }^{1,2}$, Qing Liu ${ }^{3}$, Qiongyin Wei ${ }^{4}$, Lan $\operatorname{Lin}^{4}$, Xiangqi Chen ${ }^{4}$, Dan Xue ${ }^{4}$ \\ ${ }^{1}$ Graduate School of Fujian Medical University, Fuzhou, China; ${ }^{2}$ Department of Radiation Oncology, ${ }^{3}$ Department of Oncology, ${ }^{4}$ Department of \\ Respiratory Medicine, Fujian Medical University Union Hospital, Fuzhou, China \\ Correspondence to: Xiangqi Chen; Dan Xue. Department of Respiratory Medicine, Fujian Medical University Union Hospital, No. 29, Xinquan Road, \\ Fuzhou, China. Email: chxqq2003@126.com; linty353219@vip.163.com.
}

\begin{abstract}
Hyperprogressive disease (HPD) is a phenomenon defined as extremely rapid tumor progression within a short time following immunotherapy. To date, distinguishing which subgroups may be eligible for anti-PD-1/PD-L1 treatment has presented a clinical challenge. Moreover, no sufficiently convincing biomarkers of HPD have been identified. Herein, we present two cases of cancer patients who suffered from liver metastasis before immunotherapy. A 63-year-old man presented with cough and pain in right collarbone. He was finally diagnosed as suffering from right upper lobe adenocarcinoma with cTxN3M1c and stage IVB. First-line carboplatin plus pemetrexed chemotherapy combined with sintilimab anti-PD-1 was initiated after a multi-disciplinary discussion. In the second case, a 46-year-old female was diagnosed as moderately differentiated cervical squamous cell carcinoma. Widespread recurrence 2 years after extensive total hysterectomy for early cervical carcinoma. After six cycles of first-line chemotherapy and radiotherapy, the disease progressed and new-onset liver metastasis was detected. Pembrolizumab plus abraxane was administered as second-line therapy. After the first cycle of anti-PD-1 therapy, in both cases, an extremely rapid radiological progression was observed in the liver metastases with obvious symptoms, while the primary tumor site and other metastatic lesions remained stable or shrunken. These aberrations were confirmed as HPD. The risk of HPD appears to be higher in patients with liver metastases. We believe that further research will pave the way for the discovery of more significant biomarkers of HPD.
\end{abstract}

Keywords: Hyperprogression disease; advanced cancer; liver metastasis; programmed cell death protein-1 (PD-1); immune checkpoint inhibitors

Submitted Apr 21, 2020. Accepted for publication Jul 04, 2020.

doi: 10.21037/atm-20-3928

View this article at: http://dx.doi.org/10.21037/atm-20-3928

\section{Introduction}

Immunotherapy is often used as a last resort for patients with malignant tumors, especially those with systemic metastasis. The treatment has been approved for the treatment of various cancers including melanoma, lung cancer, and Hodgkin lymphoma by FDA (1-4). The new treatment can extend the life span of patients and control tumor development to a degree. PD-1 (programmed cell death protein-1), a receptor found on the surface of activated $\mathrm{T}$ cells, binds to its ligands, which are commonly expressed in cancer cells (5); this inhibits the activation of immune cell and results in tumor immune escape $(6,7)$.
Anti-PD-1/PD-L1 immune checkpoint inhibitors can block PD-1 from binding with its ligands and restore the vitality of effector $T$ cells, thus reducing the chance of immune escape (8). The proven anti-tumor effect and good tolerability of PD-L1 antibody have led to it being widely used in clinic (9). However, the safety of anti-PD-1/PD-L1 therapy demands urgent attention.

Anti-PD-1/PD-L1 treatment has been associated with hyperprogressive disease (HPD), which refers to the phenomenon of rapid disease progression within a short time of treatment commencing. Initially described by Champiat et al., HPD has an incidence of $9 \%$ (10). It has 


\section{Page 2 of 9}

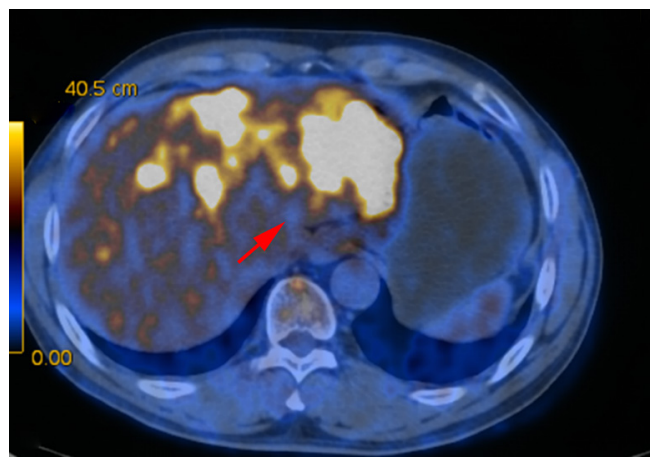

Figure 1 Positron emission tomography-computed tomography (PET-CT) imaging showed diffuse metastases lesion (indicated by red arrowhead) in liver before anti-PD-1 therapy.

been detected across various types of cancers and some subsets of patients are particularly susceptible (11). Due to the strong relationship between HPD and poor prognosis, there is an urgent need to identify the predictors and mechanisms of HPD $(11,12)$.

Herein, we present two cases with advanced cancer who experienced rapid progression of liver metastasis after the commencement of chemotherapy combined with anti-PD-1 therapy. This case study aims to explore the effects of liver metastasis on HPD.

\section{Case presentation}

All procedures performed in the cases involving human participants were in accordance with the Declaration of Helsinki (as revised in 2013). No approval by ethical committee or institutional review board was required. Written informed consent was obtained from the families of the patients for publication of the cases.

\section{Case 1}

A 63-year-old man with a history of cough and pain in right collarbone presented at our hospital. The patient had no history of smoking. Relevant examinations were carried out after admission. Chest computed tomography (CT) detected a mass in the lung. Positron emission tomographycomputed tomography (PET-CT) revealed the shadow of a lobulated mass, measuring about $36 \mathrm{~mm} \times 32 \mathrm{~mm}$, in the right upper lobe, and multiple enlargement of the right hilar, right supraclavicular, and mediastinal lymph nodes. The value of maximal standard uptake value (SUV) was 18.5 . The nature of the mass was determined by
Lin et al. HPD in patients with liver metastasis treated with ICls

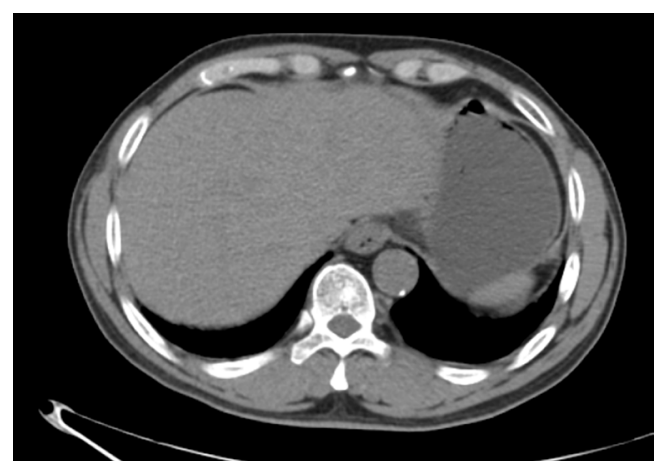

Figure 2 CT image of liver in positron emission tomographycomputed tomography (PET-CT) imaging.

tracheoscopy biopsy. Multiple nodules were detected in both lungs and regarded as intrapulmonary metastatic lesions. PET-CT also showed right clavicle metastases and liver diffuse metastases (Figure 1). No prominent solid masses were found in the liver through PET-CT imaging (Figure 2), although there were high metabolic signals in the liver. The final diagnosis was right upper lobe adenocarcinoma with cTxN3M1c and stage IVB. Gene detection showed the cancer cells were epidermal growth factor receptor (EGFR) wild type and the statuses of anaplastic lymphoma kinase (ALK) and ROS-1 were negative. The PD-L1 positive rate of the tumor cells was less than $1 \%$.

After a multi-disciplinary discussion, it was decided that the patient would undergo systemic treatment to control the disease. He underwent first-line carboplatin (AUC =5) plus pemetrexed $500 \mathrm{mg} / \mathrm{m}^{2}$ chemotherapy combined with sintilimab $200 \mathrm{mg}$ anti-PD-1, given every 3 weeks. The first cycle was completed on December 16, 2019. During hospitalization, stereotactic body radiotherapy (SBRT) was adopted to ease pain in the right collarbone, at a total dose of $8 \mathrm{~Gy}$ in a single fraction. The day after starting immune therapy, the patient developed abdominal discomfort and a poor appetite, which we evaluated as side effects of the preceding chemotherapy. By December 21, 2019, he had developed a progressively and rapidly enlarging mass in the upper left abdomen with severe persistent pain. The CT scan performed on December 23, 2019 showed the liver metastases had progressed significantly (as shown in Figure 3). For more accurate evaluation of the lesions, liver magnetic resonance imaging (MRI) was performed on December 29, 2019 and showed that the scattered metastases lesions had fused into a large mass (Figure 4). After the first cycle of treatment, the indicators 


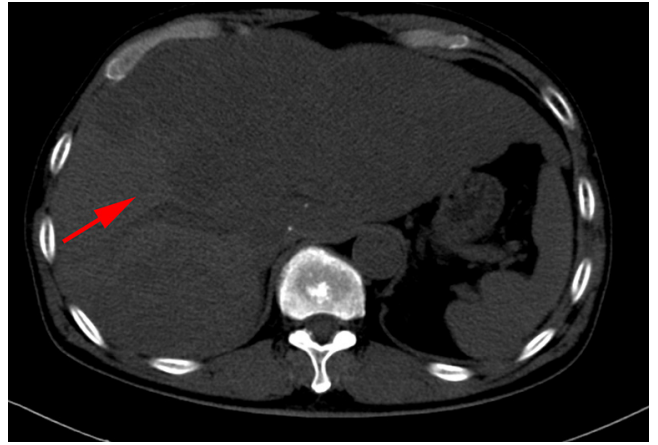

Figure 3 Abdominal computed tomography (CT) imaging performed on December 23, 2019 showed metastatic lesions (indicated by red arrowhead) in the liver after anti-PD-1 therapy.

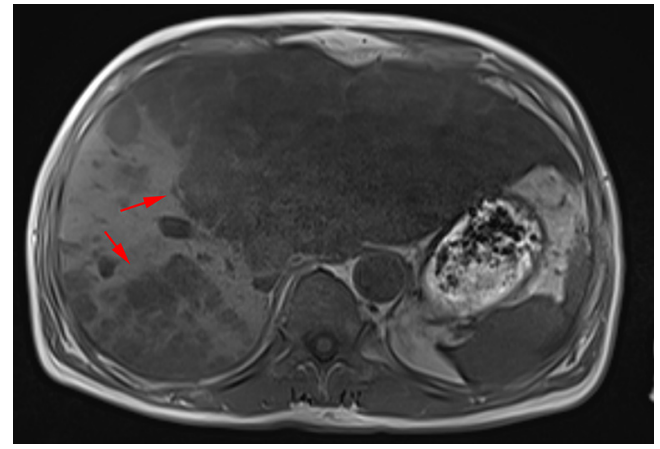

Figure 4 Magnetic resonance imaging (MRI) performed on December 29, 2019 showed metastatic lesions (indicated by red arrowheads) in the liver after anti-PD-1 therapy.

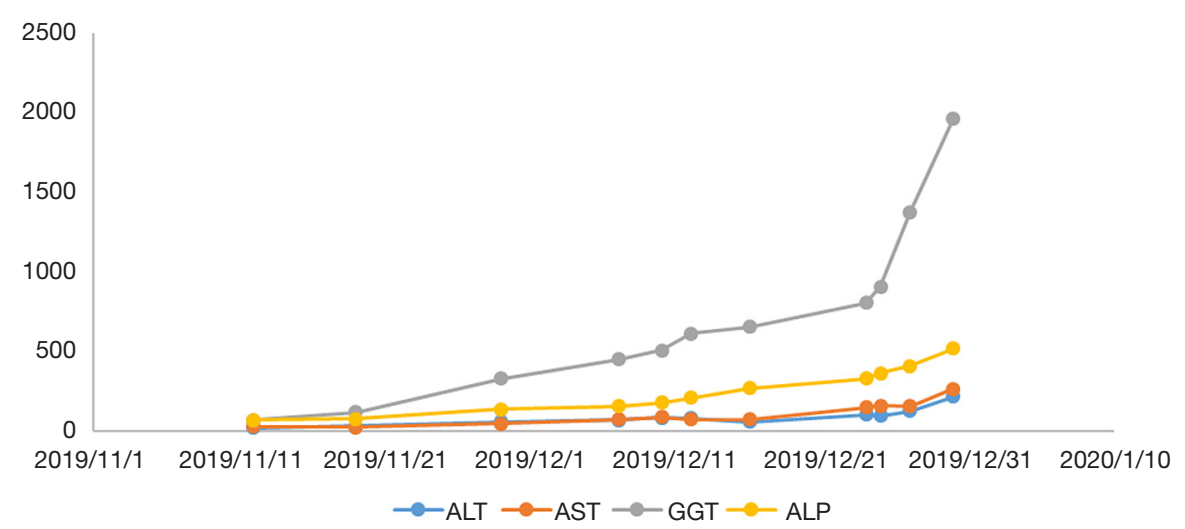

Figure 5 Changes in alanine aminotransferase (ALT), aspartate aminotransferase (AST), gamma-glutamyl transpeptidase (GGT), and alkaline phosphatase (ALP) during anti-tumor treatment.

of liver function had increased significantly (Figure 5). Pseudoprogression could be ruled out based on the patient's clinical and imaging characteristics. Despite our attempts to control the disease using the most powerful treatment possible, the patient died on January 2, 2020. The timeline of treatment administration is shown in Figure 6.

\section{Case 2}

A 46-year-old female with a history of menostaxis and heavy menstrual bleeding presented at our hospital. She had been diagnosed with moderately differentiated cervical squamous cell carcinoma in June 2017. After one cycle paclitaxel plus nedaplatin neoadjuvant chemoradiotherapy, she underwent extensive total hysterectomy combined with double-sided ovarian resection and pelvic cavity lymph node dissection in July 2017. The patient's postoperative pathological stage was IIa-IIb. As the above treatments were carried out in another hospital, other details are limited. The patient was regularly followed-up after discharge from hospital.

The patient visited our hospital with a cough in June 2019. PET-CT was performed and revealed postoperative cervical cancer with multiple metastases in the right lung, lymph nodes adjacent to the left common iliac vessels, and para-aortic lymph nodes. Gene detection showed positive PI3CA mutation status and low-grade microsatellite instability (MSI). Cisplatin plus paclitaxel was administered as first-line chemotherapy every 3 weeks. Meanwhile the metastatic lymph nodes were treated with local radiotherapy at a total dose of $45 \mathrm{~Gy} / 25 \mathrm{~F}$. After six cycles of chemotherapy and radiotherapy, CT imaging performed in January 5, 2020 revealed disease progression in all 


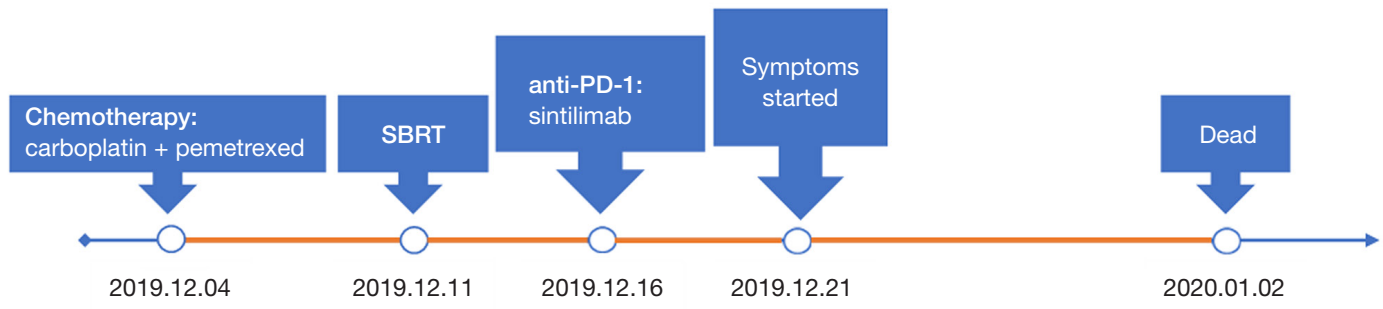

Figure 6 The timeline showing treatment administration in case 1.
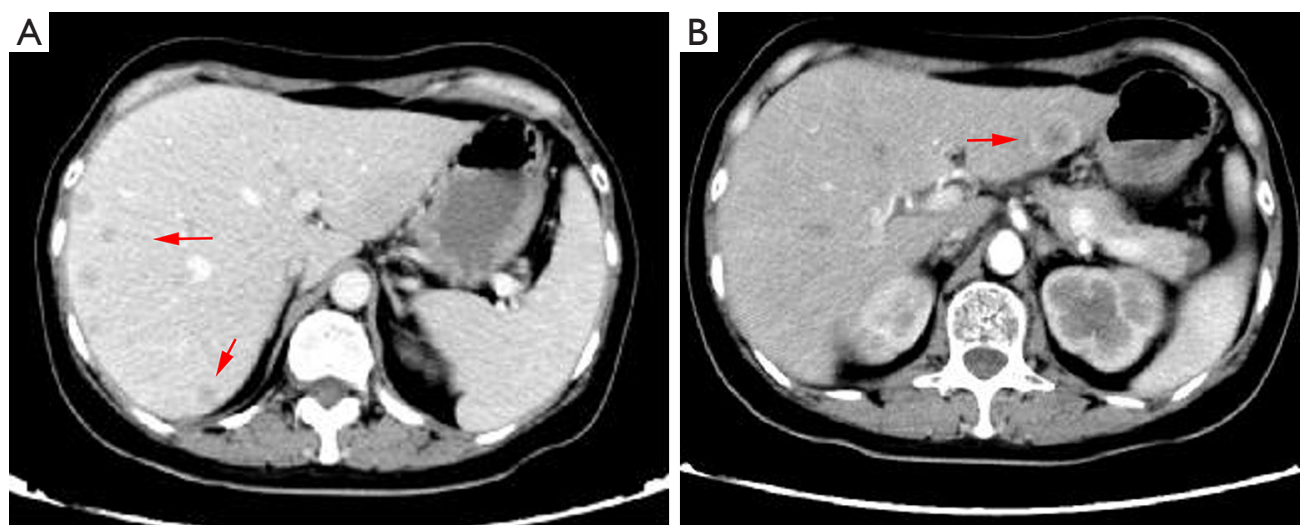

Figure 7 Abdominal computed tomography (CT) imaging performed on January 5, 2020 showed new metastatic lesions in the liver after six cycles of chemotherapy. New metastatic lesions were indicated by red arrows.

metastatic lesions and new-onset liver metastasis (Figure 7). Some of these low-density foci were diagnosed as hepatic cysts by CT imaging. Pembrolizumab plus abraxane was administered as second-line therapy every 3 weeks. The first cycle started on January 8, 2020.

From February 10, 2020, the patient progressively and rapidly developed symptoms of biliary obstruction, with extremely elevated levels of liver enzymes and bilirubin. The CT scan performed on February 25, 2020 showed that the liver metastases had progressed significantly (Figure 8), while the other metastatic lesions showed obvious shrinkage or steady state (Figures 9,10). We detected the change and evaluated it as HPD. We offered prompt treatment to control the disease, but the patient ultimately declined treatment. The timeline of treatment administration is shown in Figure 11.

\section{Discussion}

Immunotherapy combined with chemotherapy can significantly improve cellular immunity and therapeutic effectiveness in advanced cancer patients. However, the occurrence of HPD can undermine these potential benefits. When making treatment decisions, clinicians should take into account the potential risk of HPD caused by antiPD-1 therapy, and early identification of the risk factors is especially important.

Pseudoprogression and HPD have been described as two unique patterns of tumor response to immunotherapy. Recognizing the distinction between these two patterns can inform our approach to taking appropriate therapeutic measures. Pseudoprogression is characterized by enlarged tumor lesions or the presence of new lesions that have been confirmed by biopsy as abundant inflammatory cell infiltration $(13,14)$. It is followed tumor lesion shrinkage and is often considered to be associated with better treatment response and survival. The underlying mechanism behind this pattern has been described as "inflammatory flare-up" resulting from the sudden infiltration of immune cells into the tumor lesions (15).

Pseudoprogression is entirely different from HPD, the mechanism of which remains to be elucidated. A review 

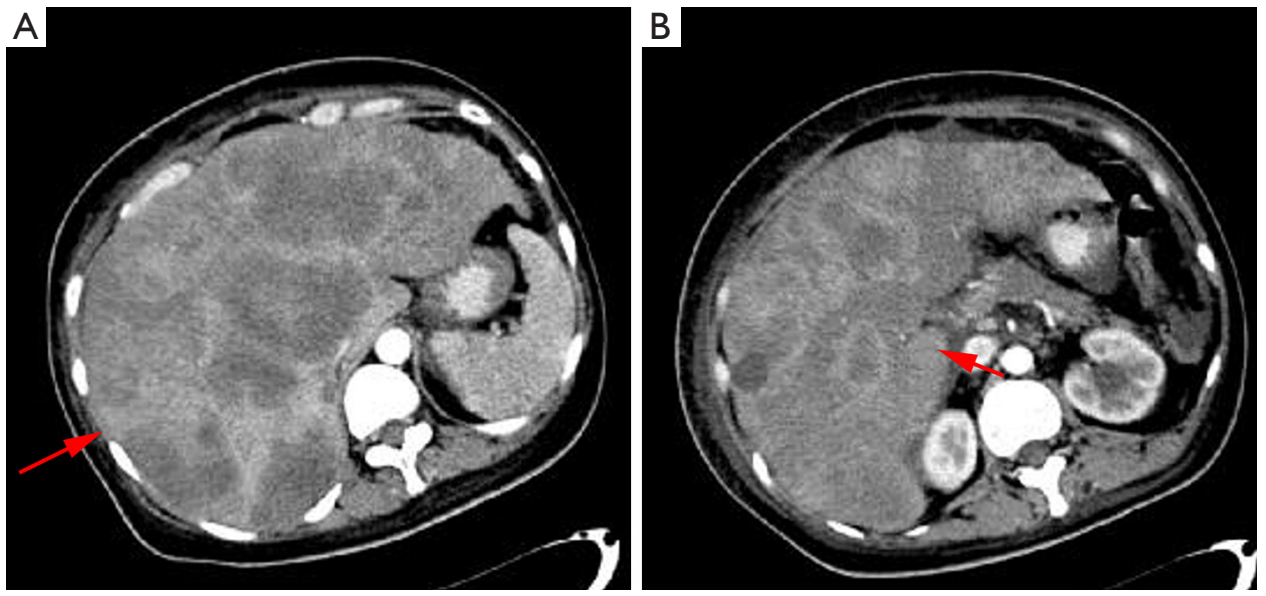

Figure 8 Abdominal computed tomography (CT) imaging performed on February 25, 2020 showed that the metastatic lesions (as pointed by red arrows) in the liver had progressed significantly after the first cycle of pembrolizumab plus abraxane.
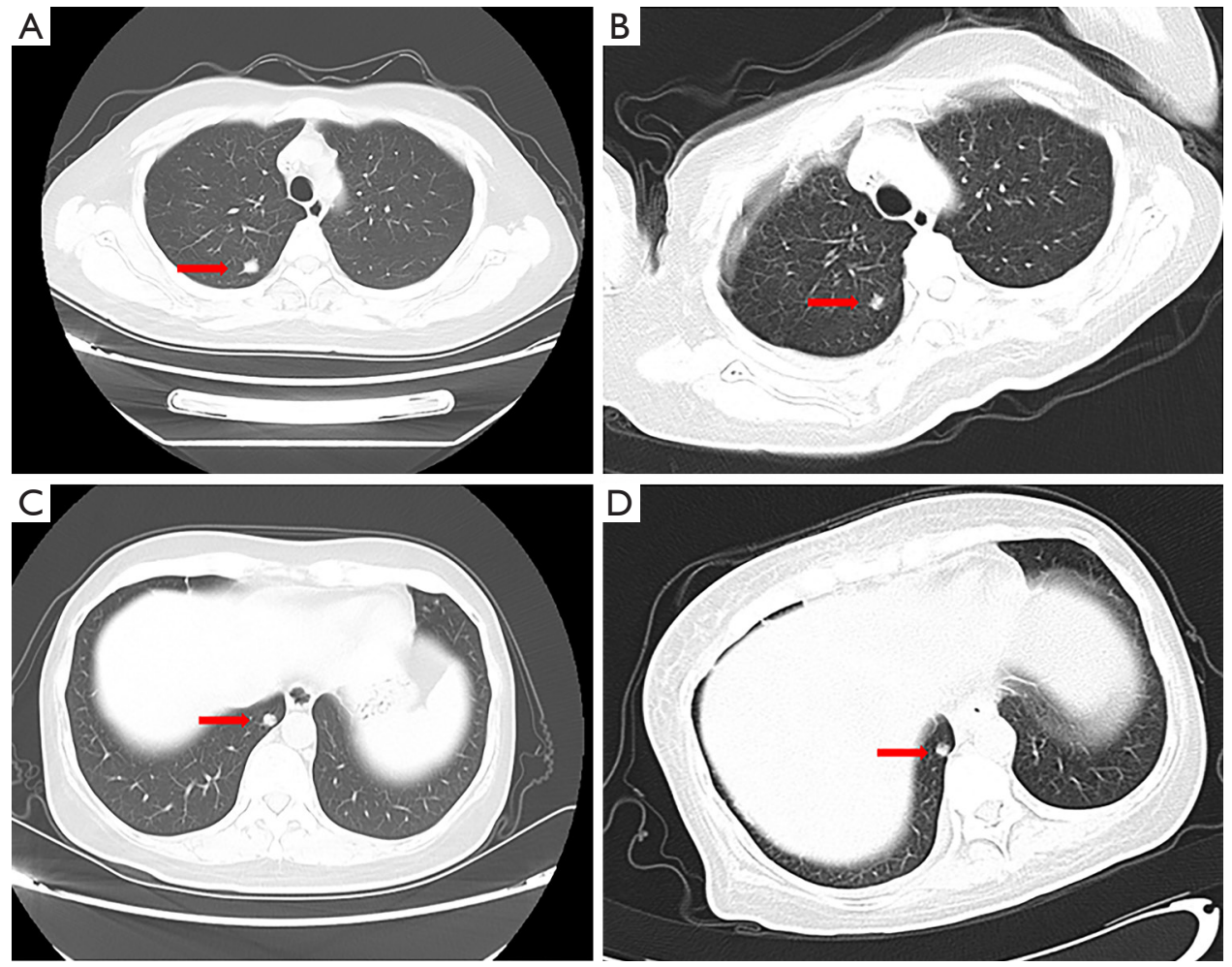

Figure 9 Chest computed tomography (CT) imaging performed on February 25, 2020 (B and D) showed lung metastatic lesions staid steady versus that was performed on January 5, 2020 (A and C). Red arrowheads: lung metastatic lesions.

of the underlying mechanisms that might cause confusion between the two conditions (16) hypothesized that the following five aspects are involved: excessive proliferation of regulatory $\mathrm{T}$ cells (Tregs); overexpression of several by-passing immune checkpoints, which causes $\mathrm{T}$ cells to become exhausted; modulation of tumor-promoting cells; exceptional inflammation via upregulation of the IL-6/IL17-neutrophil axis; and activation of oncogenes. EGFR 

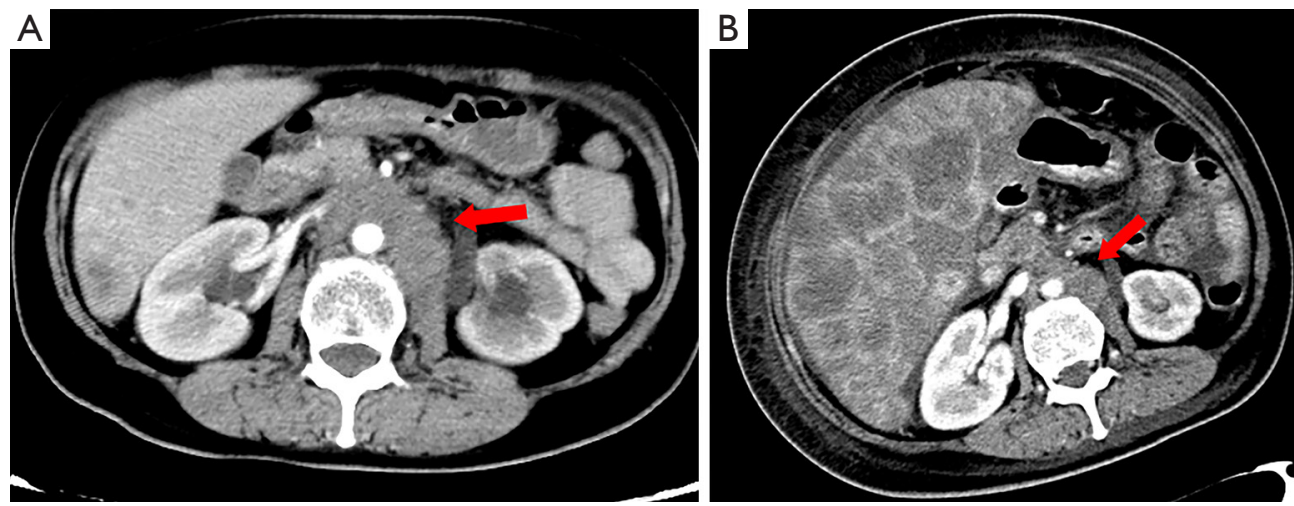

Figure 10 Abdominal computed tomography (CT) imaging performed on February 25, 2020 (B) showed obvious shrinkage in pelvic involved lymph nodes versus that was performed on January 5, 2020 (A). Red arrowheads: pelvic involved lymph nodes.

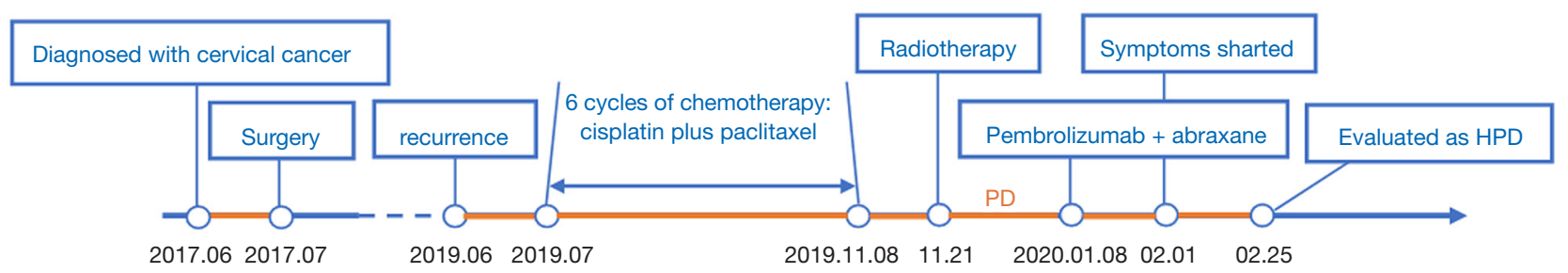

Figure 11 The timeline showing treatment administration in case 2.

mutation and amplification of MDM2 have also been associated with HPD in patients with solid tumors treated with anti-PD-1/PD-L1 therapy (11). Immune checkpoint inhibitors can upregulate MDM2 expression by stimulating CD4+ T cells to secrete IFN- $\gamma$ on a larger scale (17). The evaluated level of MDM2 mediates posttranscriptional inactivation of $\mathrm{p} 53$ by blocking the p53 transactivation domain and tagging $\mathrm{p} 53$ for ubiquitin-mediated proteasome degradation $(18,19)$. The absence or mutation of $\mathrm{p} 53$ is well known to have an important relationship with oncogenesis.

Unlike common cancer progression, HPD has its own characteristics. Ferrara and Aoki observed that HPD was much more likely to occur in patients who underwent antiPD-1/PD-L1 therapy alone than in those who received chemotherapy alone $(12,20)$. These results suggest that HPD is not simply progression caused by insensitivity to conventional chemotherapy and anti-PD-1/PD-L1 immune checkpoint therapy can contribute to its occurrence.

For the identification and evaluation of HPD, Lo Russo et al. recommended the following criteria: (I) timeto-treatment failure (TTF, defined as the time between the beginning and the discontinuation of ICI treatment)
$<2$ months; (II) a $\geq 50 \%$ increase in the sum of the major diameters of the target lesions between baseline and first radiologic evaluation; (III) the appearance of at least two new lesions in an organ already involved during the first radiologic evaluation; (IV) the involvement of a new organ detected by the first radiologic evaluation; and (V) an Eastern Cooperative Oncology Group (ECOG) performance status score of $\geq 2$ within the first two months of treatment (21). Diagnosis of HPD requires at least three of these criteria. In the two cases we reported, the patients experienced a short period of rapid progression with a TTF of less than 2 months. Although case 1 experienced no significant enlargement of the primary lung lesion, the sum of the major diameters of the target lesions increased by at least $50 \%$. In case 2 , there was obvious shrinkage in the other metastatic lesions, while the lesions in the liver experienced a sharp progression. Both patients therefore met the second criterion. Both patients also had at least two new metastasis lesions presenting in the liver. Furthermore, as the patients' digestive symptoms were severe enough to confine them to their beds, they each had an ECOG score of 4 . Based on these criteria, our judgment was confirmed. 
Interestingly, in our study, the liver metastases progressed extremely quickly while the primary lesion staid shrunken "stable disease (SD)". So does liver metastasis play an important role in the development of HPD? Liver-induced peripheral tolerance is a relatively mature theory that was first described in relation to organ transplants. Unlike other organs such as the heart and kidney, liver transplants do not depend on histocompatibility and immune suppression to prevent immunological rejection. Even more incredible is that peculiarities of the transplanted liver can spread to other organs received by the transplant recipient from the same donor, which indicates that the immune tolerance generated by liver is systemic (22-24). Some viruses can use this mechanism to evade CD8+ T cells (25). Lee et al. showed that mice with liver metastases suffered from a decrease in tumor antigen tetramer-positive CD8+ T cells, which introduces the idea that liver metastases can have an systematic effect on the host's immune system (26). And the result echoed this theory. Currently, this mechanism is under observation in confirmatory studies. Tumeh et al. found that the density of CD8+ T cells at the margin of invasive lesions was reduced in patients with liver metastasis (27). It is also reasonable to suggest that patients with liver metastases may have worse baseline physical conditions, which increases the risk of HPD. Many articles have shown liver metastases to be strongly associated with poor survival in cancer patients who receive anti-PD-1/PD-L1 therapy $(27,28)$. Indeed, some clinicians have also observed this phenomenon. Kim et al. conducted a meta-analysis of nine articles, which showed that liver metastases are strongly associated with HPD during anti-PD-1/PD-L1 therapy $(\mathrm{OR}=3.33, \mathrm{P}<0.001)$ (29). The paths of progression in the two cases dovetail with these findings in that the symptoms and indicators of the hepatobiliary system changed dramatically in a short time after anti-PD-1 therapy. Although the emergence of HPD during immunotherapy is independently related to greater metastatic burden, older age, MDM2 amplification, oncogenic EGFR mutation, and previous radiotherapy, the role of liver metastasis should also be seriously considered in the decision-making process $(10,11,30)$. Further research is needed to validate our findings and to explore potential predictors of HPD.

\section{Conclusions}

Although immune checkpoint inhibitors are thought to be associated with survival benefit in advanced cancer patients, the ability to accurately predict and prevent immunotherapy-related HPD is crucial. By reviewing and analyzing the cases above, we observed a strong association between HPD during anti-PD-1/PD-L1 and liver metastasis. This reinforces the need to carefully select patients for immunotherapy according to specific subsets. We predict that useful biomarkers and predictors will be identified in the future and will become a hot topic in managing cancer.

\section{Acknowledgments}

Funding: This study was supported by Joint Funds for the Innovation of Science and Technology, Fujian, China [grant number 2017Y9031], and by the Science Technology Innovation Joint Project Foundation of Fujian Province (grant no. 2016Y9028 and 2018Y9038).

\section{Footnote}

Conflicts of Interest: All authors have completed the ICMJE uniform disclosure form (available at http://dx.doi. org/10.21037/atm-20-3928). The authors have no conflicts of interest to declare.

Ethical Statement: The authors are accountable for all aspects of the work in ensuring that questions related to the accuracy or integrity of any part of the work are appropriately investigated and resolved. All procedures performed in the cases involving human participants were in accordance with the Declaration of Helsinki (as revised in 2013). No approval by ethical committee or institutional review board was required. Written informed consent was obtained from the families of the patients for publication of the cases.

Open Access Statement: This is an Open Access article distributed in accordance with the Creative Commons Attribution-NonCommercial-NoDerivs 4.0 International License (CC BY-NC-ND 4.0), which permits the noncommercial replication and distribution of the article with the strict proviso that no changes or edits are made and the original work is properly cited (including links to both the formal publication through the relevant DOI and the license). See: https://creativecommons.org/licenses/by-nc-nd/4.0/.

\section{References}

1. Robert C, Long GV, Brady B, et al. Nivolumab in 
previously untreated melanoma without BRAF mutation. N Engl J Med 2015;372:320-30.

2. Robert C, Schachter J, Long GV, et al. Pembrolizumab versus Ipilimumab in Advanced Melanoma. N Engl J Med 2015;372:2521-32.

3. Borghaei H, Paz-Ares L, Horn L, et al. Nivolumab versus Docetaxel in Advanced Nonsquamous Non-Small-Cell Lung Cancer. N Engl J Med 2015;373:1627-39.

4. Ansell SM, Lesokhin AM, Borrello I, et al. PD-1 blockade with nivolumab in relapsed or refractory Hodgkin's lymphoma. N Engl J Med 2015;372:311-9.

5. Pardoll DM. The blockade of immune checkpoints in cancer immunotherapy. Nat Rev Cancer 2012;12:252-64.

6. Berger JR. PD-1 inhibition: a novel approach to the treatment of progressive multifocal leukoencephalopathy. Ann Transl Med 2019;7:S281.

7. Jove M, Vilariño N, Nadal E. Impact of baseline steroids on efficacy of programmed cell death-1 (PD-1) and programmed death-ligand 1 (PD-L1) blockade in patients with advanced non-small cell lung cancer. Transl Lung Cancer Res 2019;8:S364-8.

8. Okazaki T, Honjo T. PD-1 and PD-1 ligands: from discovery to clinical application. Int Immunol 2007;19:813-24.

9. Chen R, Tao Y, Xu X, et al. The efficacy and safety of nivolumab, pembrolizumab, and atezolizumab in treatment of advanced non-small cell lung cancer. Discov Med 2018;26:155-66.

10. Champiat S, Dercle L, Ammari S, et al. Hyperprogressive Disease Is a New Pattern of Progression in Cancer Patients Treated by Anti-PD-1/PD-L1. Clin Cancer Res 2017;23:1920-8.

11. Kato S, Goodman A, Walavalkar V, et al. Hyperprogressors after Immunotherapy: Analysis of Genomic Alterations Associated with Accelerated Growth Rate. Clin Cancer Res 2017;23:4242-50.

12. Ferrara R, Mezquita L, Texier M, et al. Hyperprogressive Disease in Patients With Advanced Non-Small Cell Lung Cancer Treated With PD-1/PD-L1 Inhibitors or With Single-Agent Chemotherapy. JAMA Oncol 2018;4:1543-52.

13. Wolchok JD, Hoos A, O'Day S, et al. Guidelines for the evaluation of immune therapy activity in solid tumors: immune-related response criteria. Clin Cancer Res 2009; 15:7412-20.

14. Wang Q, Gao J, Wu X. Pseudoprogression and hyperprogression after checkpoint blockade. Int Immunopharmacol 2018;58:125-35.
15. Solinas C, Porcu M, Hlavata Z, et al. Critical features and challenges associated with imaging in patients undergoing cancer immunotherapy. Crit Rev Oncol Hematol 2017;120:13-21.

16. Champiat S, Ferrara R, Massard C, et al. Hyperprogressive disease: recognizing a novel pattern to improve patient management. Nat Rev Clin Oncol 2018;15:748-62.

17. Peng $W$, Liu C, Xu C, et al. PD-1 blockade enhances T-cell migration to tumors by elevating IFN-gamma inducible chemokines. Cancer Res 2012;72:5209-18.

18. Vassilev LT, Vu BT, Graves B, et al. In vivo activation of the p53 pathway by small-molecule antagonists of MDM2. Science 2004;303:844-8.

19. Wong DJ, Lee J, Choo SP, et al. Hyperprogressive disease in hepatocellular carcinoma with immune checkpoint inhibitor use: a case series. Immunotherapy 2019;11:167-75.

20. Aoki M, Shoji H, Nagashima K, et al. Hyperprogressive disease during nivolumab or irinotecan treatment in patients with advanced gastric cancer. ESMO Open 2019;4:e000488.

21. Lo Russo G, Moro M, Sommariva M, et al. Antibody$\mathrm{Fc} / \mathrm{FcR}$ Interaction on Macrophages as a Mechanism for Hyperprogressive Disease in Non-small Cell Lung Cancer Subsequent to PD-1/PD-L1 Blockade. Clin Cancer Res 2019;25:989-99.

22. Calne RY. Immunological tolerance--the liver effect. Immunol Rev 2000;174:280-2.

23. Calne RY, Sells RA, Pena JR, et al. Induction of immunological tolerance by porcine liver allografts. Nature 1969;223:472-6.

24. Cantor HM, Dumont AE. Hepatic suppression of sensitization to antigen absorbed into the portal system. Nature 1967;215:744-5.

25. Tu Z, Pierce RH, Kurtis J, et al. Hepatitis C virus core protein subverts the antiviral activities of human Kupffer cells. Gastroenterology 2010;138:305-14.

26. Lee J, Mehdizadeh S, Tsai K, et al. Immunological insights into liver metastasis associated resistance to checkpoint blockade cancer immunotherapy. J Immunol 2018;200.

27. Tumeh PC, Hellmann MD, Hamid O, et al. Liver Metastasis and Treatment Outcome with Anti-PD-1 Monoclonal Antibody in Patients with Melanoma and NSCLC. Cancer Immunol Res 2017;5:417-24.

28. Funazo T, Nomizo T, Kim YH. Liver Metastasis Is Associated with Poor Progression-Free Survival in 
Patients with Non-Small Cell Lung Cancer Treated with Nivolumab. J Thorac Oncol 2017;12:e140-e141.

29. Kim JY, Lee KH, Kang J, et al. Hyperprogressive Disease during Anti-PD-1 (PDCD1)/PD-L1 (CD274) Therapy: A Systematic Review and Meta-Analysis. Cancers (Basel)

Cite this article as: Lin Z, Liu Q, Wei Q, Lin L, Chen X, Xue D. Hyperprogressive disease in advanced cancer patients with liver metastasis treated with PD-1 inhibitors: two case reports. Ann Transl Med 2020;8(17):1100. doi: 10.21037/atm20-3928
2019. doi: 10.3390/cancers11111699.

30. Saâda-Bouzid E, Defaucheux C, Karabajakian A, et al. Hyperprogression during anti-PD-1/PD-L1 therapy in patients with recurrent and/or metastatic head and neck squamous cell carcinoma. Ann Oncol 2017;28:1605-11. 